\title{
Effect of changing polarity of graphite tool/ Hadfield steel workpiece couple on machining performances in die sinking EDM
}

\author{
Hacı Bekir Özerkan ${ }^{1, *}$ \\ ${ }^{1}$ Gazi University Vocational School of Technical Sciences, Department of Machine and Metal Technology, 06374 Ankara, Turkey
}

\begin{abstract}
In this study, machining performance ouput parameters such as machined surface roughness (SR), material removal rate (MRR), tool wear rate (TWR), were experimentally examined and analyzed with the diversifying and changing machining parameters in (EDM). The processing parameters (input par.) of this research are stated as tool material, peak current (I), pulse duration $\left(t_{\text {on }}\right)$ and pulse interval $\left(t_{\text {off }}\right)$. The experimental machinings were put into practice by using Hadfield steel workpiece (prismatic) and cylindrical graphite electrodes with kerosene dielectric at different machining current, polarity and pulse time settings. The experiments have shown that the type of tool material, polarity (direct polarity forms higher MRR, SR and TWR), current (high current lowers TWR and enhances MRR, TWR) and pulse on time ( $t_{\text {on }}=48 \square$ s is critical threshold value for MRR and TWR) were influential on machining performance in electrical discharge machining.
\end{abstract}

\section{Introduction}

The electrical discharge machining (EDM) is widely applied, practiced and important manufacturing technique, in die and mold making industry to fabricate multifaceted and three dimensional complicated geometries made of electrical conductive materials carry out roughy and/or mirror-like finishing operations optionally [1]. This technique process was predicate on removing material from an untreated raw metal part by way of sequential random electrical discharges between tool and the work piece in dielectric environment [2]. The electrode is approached to the work piece until the initial former sparks occur since therefore the operating voltage creates the required and sufficient energy to ionize the dielectric fluid [3]. In this technique, main EDM performance parameters are known as electrode material, work piece materials, dielectric and the electrical parameters consists of polarity, pulse on/off time, current and voltage. Ho and Newman remarked that EDM research topics could be arranged into three basic titles, these are measurement and determining of machining performance under different machining conditions, the influences of machining inputs and designation and fabricating of tools. They educed that the main and effective machining performance outputs are attributed to the MRR, tool wear and machined surface quality [1]. One of the most important electrical parameters of EDM is polarity [4].For briefly enlightening of electrical erosion mentality, and the morphological, metallurgical and textural ultimate surface characteristics, it's always necessary to study the effect of polarity which is defined as reverse polarity (electrode positive) and straight polarity (electrode negative) $[5,6]$.

From the previous and published literature, main machining performance characteristics (MRR, TWR, SR) of difficult to cut Hadfield steel are still infrequent in die sinker EDM with graphite tools. Hence, it's believed that more studies on difficult to hard metals could be optimize the process and allow more feasible ways to increase the overall productivity of machinability by using graphite electrodes. The main purpose of this experimental research is to obtain the effect of machining outputs such as MRR, RWR and SR $\left(R_{a}\right)$. And also this paper describes the wear behaviour of graphite tools with both polarity cases when machining Hadfield steel. The results will be utilized to choose optimal tool and work material arrangement.

\section{Experimental design and procedure}

The experimental machining tests were performed on Furkan die- sinking EDM (Fig. 1)which is energized by a 100 A iso-pulse type generator. Commercial pure kerosene was selected and used as dielectric during the experimental machinings and lateral flushing system was used to remove metal debris away.

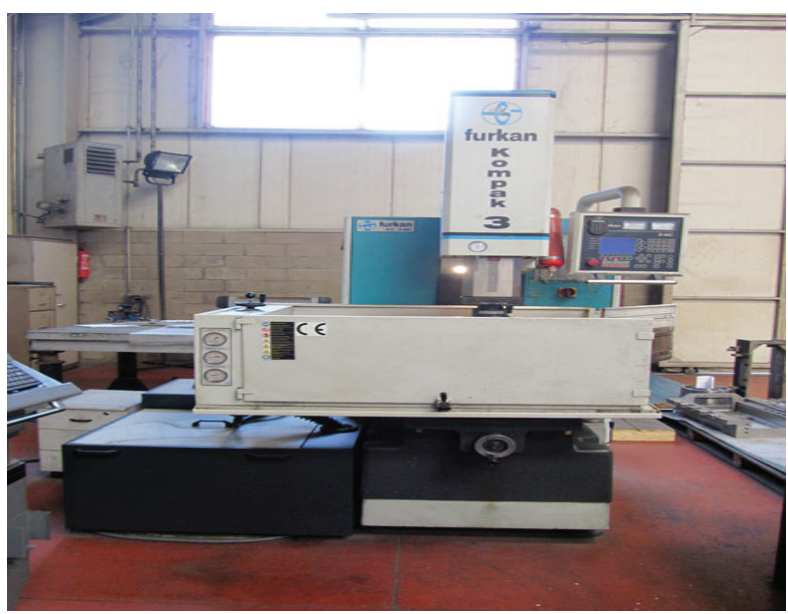

Fig. 1. ZNC Die-sinker EDM machine used in experiments.

\footnotetext{
* Corresponding author: ozerkan@gazi.edu.tr
} 
Table 1 depicts the chemical composition of the Hadfield steel and the dimensions of the prismatic workpiece samples are $30 \mathrm{mmX} 40 \mathrm{mmX} 10 \mathrm{~mm}$. Both workpiece surfaces were ground.

Table 1. Hadfield steel's chemical composition

\begin{tabular}{|c|c|c|c|}
\hline \multicolumn{4}{|c|}{ Hadfield Steel } \\
\hline $\mathbf{C}$ & $\mathbf{S i}$ & $\mathbf{M n}$ & $\mathbf{C r}$ \\
\hline 1,08 & 0,62 & 13,6 & 0,72 \\
\hline $\mathbf{M o}$ & $\mathbf{N i}$ & $\mathbf{C u}$ & $\mathbf{F e}$ \\
\hline 0,26 & 0,3 & 0,18 & 83,2 \\
\hline
\end{tabular}

It's known that the first rule of choosing the tool material is the internal structure, toughness and hardness of workpiece materials. In EDM of steels; copper, copper-tungsten and graphite electrodes are most widely used. Due to the difficult cutting of Hadfield steel with conventional methods, hoping that we can get good results by the best conductive graphite. The electrodes of $20 \mathrm{~mm}$ diameter were used for machining Hadfield steel machinability experiments. The physical properties of graphite and in order to compare to copper are given in Table 2.

Table 2. Physical properties and comparision of graphite and copper

\begin{tabular}{|l|c|c|}
\hline & Graphite & Copper \\
\hline Density $\left(\mathrm{g} / \mathrm{cm}^{3}\right)$ & 1,8111 & 8,904 \\
\hline Melting point $\left({ }^{\circ} \mathrm{C}\right)$ & 3350 & 1083 \\
\hline $\begin{array}{l}\text { Electrical resistivity } \\
(\Omega . \mathrm{m})\end{array}$ & $2.5 \times 10^{-6} \sim 5 \times 10^{-6}$ & $1.68 \times 10^{-8}$ \\
\hline Hardness & HB 10 & HB 100 \\
\hline
\end{tabular}

Pulse on time of $3,6,12,24,48,99,201 \mu \mathrm{s}$, pulse off time of $3,6,12,24 \mu \mathrm{s}$, discharge currents of $6,12.5$, 25, $50 \mathrm{~A}$, and lateral flushing pressures of 0.5 bar were used. The other machining parameters; flushing pressure, an open circuit voltage of $80 \mathrm{~V}$, machining depth of 1 $\mathrm{mm}$ were kept stationary during the machinings. In this study, 28 different machining with reverse polarity (electrode positive) and 28 different machining with direct (straight) polarity (electrode negative) were experimentalized. Each experiment under same machining condition was carried out twice and average value of the two results was used. For obtaining the lowest TWR, highest MRR and desired SR, the effects of $I(A), t_{\text {on }}(\mu s)$ and polarity exchange were investigated and evaluated. Surface roughness measurements of machined area were performed by portable TR200 stylus device. Cut-off and evaluation length were selected from standard tables as $0.8 \mathrm{~mm}$ and $4 \mathrm{~mm}$, respectively. Ra,
$\mathrm{Rz}(\mathrm{DIN})$ and $\mathrm{Sm}$ roughness parameters were measured and recorded simultaneously. Five measurements were taken from five different zones of machined area and Ra is used for composing SR assesment graphics. The five SR measurements averaged and used in the study.

\section{Results and discussion}

The main machining performance outputs namely as; material removal rate (MRR- $\left.\mathrm{mm}^{3} / \mathrm{min}\right)$, relative wear ratio (\%) and surface roughness $\left(\mathrm{R}_{\mathrm{a}}-\mu \mathrm{m}\right)$ specified and evaluated according to the selected and studied machining performance inputs such as type of tool polarity, machining current and pulse-on time. MRR depicts the volume of removed workpiece per machining time. Eroding of both tool and workpiece is known as the fundamental law of EDM. Electrode wear rate (TWR) is defined as the ratio of weared weight of the tool to the machining time. And also relative electrode wear $(\%)$ is expressed as the percentage ratio of TWR to the eroded material volume from the work piece (MRR).

Thermo-physical features of tool material specify the talent to machining energy of the EDM and remove metals. For generating a spark, peak current is flowed through the gap after dielectric is broken down. At this point, electrons runaway from the tool for coming into collison with molecules of the dielectric fluid. Hereby dielectric is vaporized and energy plasma channel is generated permitting the spark to materialise and embody. However, graphite tools are able to emit deionizer electrons at much lower temperatures and the time required to form the energy channel is considerably less. Consequently graphite starts forming spark more fastly, intensely and strongly, resulting in explicitly higher metal removal rates. Figure 2 shows the effect of polarity, $t_{\text {on }}$ and I variaties on MRR. It is seen obviously that MRR increases with the increase in current with both reverse polarity and direct polarity. Also the inclination of the curves clarifies that MRR increases meaningfully with the increase in current with direct polarity as compared to reverse polarity.

Pulse duration-Material removal rate graphic

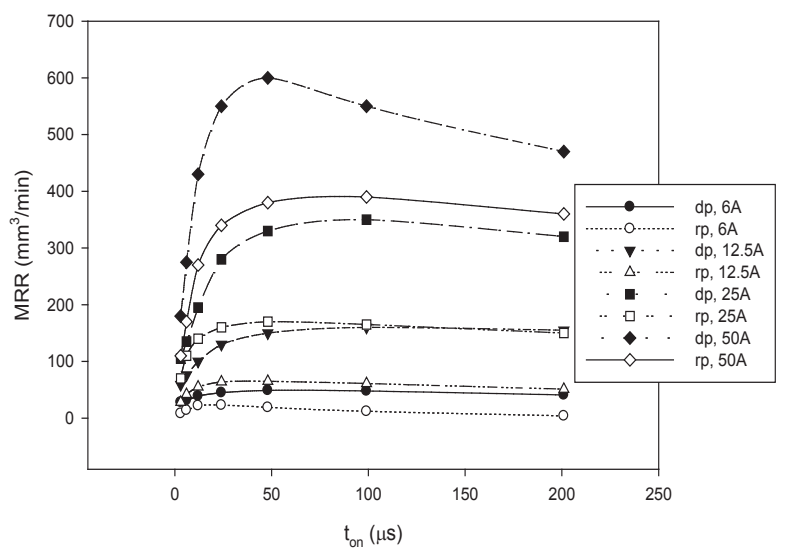

Fig. 2. $M R R\left(\mathrm{~mm}^{3} / \mathrm{min}\right)-t_{o n}(\mu \mathrm{s})$ variation. 
With an increase in current the discharge spark energy increases providing higher MRR. In direct polarity case more positively charged ions are emitted from the tools which strike on the work piece. Due to higher mass the momentum of these positively charged ions is greater than that of negatively charged electrons resulting in more eroded material from the workpiece in comparison with the electrodes. Maximum MRR is obtained at 50 amps current with negative polarity. And also the effect of pulse-on time on MRR is shown in Figure 2. MRR is increasing stably with increase in the level of the pulse-on time and apparent that longer than $48 \mu$ s pulse durations MRR decreases due to the bad, unstable, unbalanced spark discharges because of inadequate and short pulse-off times. Sparking energy is related with the MRR and directly proportional to the pulse on time $\left(\mathrm{t}_{\mathrm{on}}\right)$. However, alonger pulse duration forms and brings on uneffective long arcing that increases dielectric temperature and reduces MRR.

Pulse duration-Relative wear ratio graphic

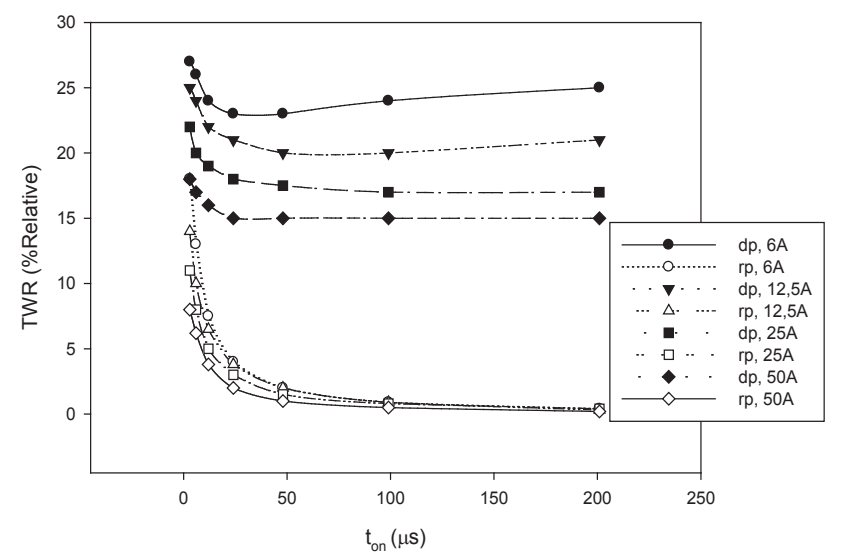

Fig. 3 TWR (\% Relative $)-t_{\text {on }}(\mu \mathrm{s})$ variation.

Fig. 3 illustrates the variation of relative wear according to pulse on time and current. There is a clear decrease in relative wear (\%) with increase in pulse on time from $(3-48 \mu \mathrm{s})$ as tool wear rate decrease and material removal rate increases in both polarity conditions. TWR decreases with $t_{\text {on }}$ time continuously in a non- linear manner. But increase in $t_{\text {on }}$ time from 48$201 \mu \mathrm{s}$ is provided moderate decrease in tool wear. Reduction in tool wear may be attributed to spark energy is directly proportional with $t_{\text {on }}$. So that discharge energy increases with increase in $t_{\text {on }}$ but after definite $t_{\text {on }}$ leads to spreading of the discharges. By the reason of this spreading reduction of heat transfer to the tool and increasing of heat transfer the work piece and dielectric occurs which leads to less tool wear and high material removal rate. And also additionally, when pulse duration and cuurent increases relative electrode wear decreases because of graphite tool composes of extra carbon element right along with carbon layer composes from deionization of kerosene adhered the machining area of electrode tool and becomes thick enough. These two affects prevent and reduce the further tool wear and also relative tool wear, as a consequence of that at lower pulse duration the relative electrode wear is more and at higher pulse duration it would be lowered.

And also from Figure 3 it is also seen that as the current increases from low to medium-high levels respectively, the relative electrode wear diminishes. Graphite, can be used on high currents without much loss and deformation in reverse polarization.

At higher $t_{\text {on }}$ carbon layer becomes more thicker and it inhibits tool wear and thus at $200 \mu$ s pulse duration the value of relative electrode wear decreases majorly as compared to the value at medium current level. So that increasing of current at low pulse duration levels relative electrode wear (\%) decreases. And also it is seen that relative electrode wear curves for direct and reverse polarity track the same decreasing trend. Lower relative electrode wear is achieved with the reverse polarity at all current and pulse duration values. It is known that at reverse polarity $40 \%$ thermal energy is generated at anode and $25 \%$ thermal energy is generated at cathode (Xia,et al.) [7] and its opposite is true for direct polarity ( $25 \%$ heat is generated at work piece and $40 \%$ heat is generated at tool). Lowest relative electrode wear is achieved at high current at high pulse duration with direct polarity and its value is $0.2 \%$. During the experiments ratio of relative electrode wear (TWR at reverse polarity/ TWR at direct polarity) ranges from 1.5-75 on an average reverse polarity gives 3.7-5.1 times more relative electrode wear as compared to direct polarity. Minimum TWR is obtained at 50 amps current with reverse polarity. Tool wear is dependent on intensity of spark enegy and tool materials type. High electrical conductivitity and melting point (difficult to cut materials) of any metal behaves more resistable to wear because of requiring large amount of energy to melt. Typically used tool materials perform different wearing behaviours with different workpiece materials and polarity combinations. Consequently reverse polarity is more suitable for getting lower relative graphite wears. The surface roughness results at direct and reverse polarity are shown in Figure 4 at different current and pulse duration levels. It is seen obviously that $\mathrm{Ra}$ increases with the increase of pulse duration and current despite this at direct polarity case $R_{a}$ values are higher than the reverse polarity condition. This tendency is opposite to the general trend of increasing surface roughness with the reverse polarity. This observation is supported by Han, et al. [8] and Lin et al. $[9,10]$. This effect may be because of higher electrical conductivity of graphite electrodes which produces high intensity impact spark forces that strikes on the work piece with the increase of $I$ and $t_{\text {on }}$ at direct polarity. Thus this higher impact forces cavitated and formed more deep and wide discharge craters. Consequently the surface integrity improves as the discharge energy and spark impact force decreases but with graphite tool and direct polarity is occured opposite situation that produces rougher surfaces. From the curves of surface roughness at reverse polarity it is seen that as the pulse duration and current increases the surface roughness decreases. This surface structure is harmonic and agreeable at both polarity with the tool wear graphs. Generation of large craters due to higher sparking energy and also electrical 
conductivity and melting point of tool material ends up with higher tool wear and big roughness values with higher $t_{o n}$ and I. Thus, for achieving better surface finishings it necessitates low $I$ and $t_{\text {on }}$ with graphite tools at reverse polarity. From the experimental results it is seen that direct polarity gives almost $0.8-1.8$ times higher surface roughness as compared to reverse polarity.

The results show that direct polarity ( - tool) is more acceptable, attractive and effective about that MRR is higher, in response to this TWR is lower when using positive tool. It can not clearly say any better performance obtained with tool/workpiece couple as cathode or anode. Direct polarity is suitable for both roughing operations. Good MRR could be achieved in rough machining with negative polarity tools. MRR is $25-81 \%$ higher than for machining with positive tool. So that with direct polarity metal removal rate and tool wear is higher as compared to reverse polarity with graphite tools. Polarity is very important for MRR, electrode wear and surface roughness.

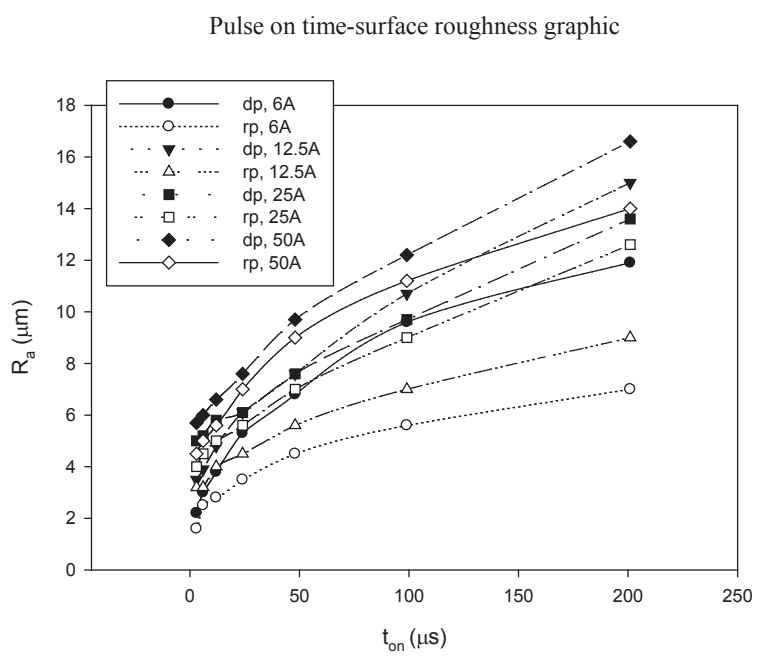

Fig. 4. $\mathrm{R}_{\mathrm{a}}(\mu \mathrm{m})-\mathrm{t}_{\mathrm{on}}(\mu \mathrm{s})$ variation.

\section{Conclusion}

An inclusive experimental study has been carried out for researching the influences of main processing parameters on optimal shaping performance of Hadfield steel in EDM. The machining inputs are tool material, polarity and pulse on time, machining outputs are MRR, TWR (\%) and surface roughness. The succinct, brief observations may be summarized from this study.

- MRR increases together with both direct and reverse polarity associated with increasings in current. The slope of the curve shows that MRR increases drastically and progressively in full value current increasings with direct polarity as compared to reverse polarity. It's explicit that longer than $48 \square$ (can be accepted as threshold value) pulse durations MRR decreases due to the bad, unstable, unbalanced sparks.

- There is an evidence decrease in relative wear (\%) with increase in pulse on time from (3-48 $\mu \mathrm{s})$ as TWR decrease and lower relative TWR (\%) is achieved with the reverse polarity at all current and pulse on time values. Furthermore machining current increasings of at low pulse on time levels relative electrode wear (\%) decreases exaggeratively. Minimum TWR is obtained at maximum current with reverse polarity.

- The effect of polarity was interesting by means of reverse polarity produced shallow and small craters however direct polarity led to larger craters. Roughness of machined surfaces increases with the increase in current and ton with both direct and reverse polarity.

It is important the type of material to be processed, higher power levels and reverse polarity must be used for difficult to cut hard metals with conventional machining processes. The working polarity plays an important role effecting the material removal rate and the properties of the machined surface. Choosing the right parameters is critical for different electrode and workpiece materials for successful operations.

\section{References}

1. K.H. Ho, S.T. Newman, Int. J. of Machine Tools \& Man. 43 (2003)

2. C.J. Luis, I. Puertas, G. Villa, J. of Materials Processing Tech. 164-165: 889-896 (2005)

3. B. Bojorquez, R.T. Marloth. O.S. Es-Said, Eng. Failure Analysis, 9 (2002)

4. H. El-Hofy, Advanced Machining Processes: Nontraditional and Hybrid Machining Processes, (McGraw-Hill Companies, 2005)

5. C.H. Che Haron, et al., Journal of Materials Processing Technology 87 (2001)

6. C. Cogun, S. Akaslan, KSME International Journal. 16 (2002)

7. H. Xia, M. Kunieda, N. Nishiwaki, Int. J. of Electrical Mach., 1 (1996)

8. F. Han, Y. Yamada, T. Kawakami, M. Kunieda, Precision Engineering 30 (2006)

9. Y.C. Lin, B.H. Yan, F.Y. Huang, Int. J. of Adv.Man. Tech. 18 (2001a)

10. Y.C. Lin, B.H. Yan, F.Y. Huang, J.1 of Mat. Processing Tech. 115 (2001b) 